UW Biostatistics Working Paper Series

$1-30-2010$

\title{
Robustness of approaches to ROC curve modeling under misspecification of the underlying probability model
}

Sean Devlin

University of Washington - Seattle Campus, devlin@u.washington.edu

Elizabeth Thomas

University of Washington, lizt@u.washington.edu

Scott S. Emerson

University of Washington, semerson@u.washington.edu

\section{Suggested Citation}

Devlin, Sean ; Thomas, Elizabeth; and Emerson, Scott S., "Robustness of approaches to ROC curve modeling under misspecification of the underlying probability model" (January 2010). UW Biostatistics Working Paper Series. Working Paper 355.

http://biostats.bepress.com/uwbiostat/paper355

This working paper is hosted by The Berkeley Electronic Press (bepress) and may not be commercially reproduced without the permission of the copyright holder.

Copyright (c) 2011 by the authors 


\section{Introduction}

A continuous medical test (marker) may be used to discriminate diseased individuals from their healthy counterparts. Without loss of generality, assume the marker tends to be higher among individuals with the disease. A specified threshold of the test above which an individual is classified as diseased generates a value for the true positive rate (sensitivity) and false positive rate (1-specificity). The receiver operating characteristic (ROC) curve is a tool to show the relationship between the true positive rate and false positive rate as the threshold of the test is varied. Mathematically we can write this as $R O C(t)=S_{D}\left(S_{\bar{D}}^{-1}(t)\right)$, where $S_{D}$ and $S_{\bar{D}}$ are the survival functions in the diseased and healthy populations, respectively. A very low threshold correctly classifies the majority of diseased cases (high sensitivity) but may incorrectly classify the majority of healthy individuals (low specificity). Similarly, a very high threshold has low sensitivity and high specificity.

As an example CA-125 and CA-19-9 are two markers used for identifying individuals with pancreatic cancer (Pepe 2003). A comparison of the two respective ROC curves can help determine which marker is able to better discriminate individuals with pancreatic cancer from the general healthy population.

Various statistical regression methods have been developed to contrast the discriminatory ability of different markers across covariate groups. Pepe (1998) distinguished three methods for ROC analysis, focusing on modeling either the marker distribution, a summary measure of the ROC curve such as area under the curve (AUC), or the ROC curve directly. Early regression methods included a full parametric model, where the unknown parameter describing the marker distribution in the healthy and diseased populations was of finite dimension, and the ROC curve was similarly defined by a finite-dimensional parameter. Fully parametric models previous described for this approach include binormal, logistic and negative exponential (Zweig and Campbell 1993). In an attempt to relax the strong assumptions of the fully parametric approach, one semi-parametric approach focuses on modeling a marker's 
survival function in both populations. After specifying these two distributions, a family of ROC curves are induced. As an example, a location-scale family could be specified for each survival function, so the marker is a shift from a common unknown function. This approach allows for interpretable covariate effects on the ROC curve, induced through the separate estimated effects for healthy and disease markers. But this specification may induce a family of ROC curves that remains dependent on this unknown function, and therefore be infinite dimensional: parameter estimates by themselves may not provide information on the actual shape of the ROC curve.

To avoid this issue of infinite dimensionality, a second semi-parametric approach, ROCGLM, focuses on modeling the ROC curve directly (Pepe 2003). This method specifies a finite dimensional parametric family of ROC curves that induce a semi-parametric model of marker distributions, which may or may not correspond to a semi-parametric model commonly used in other statistical applications. Covariates can be flexibly estimated at either the marker level, similar to the location-scale family, or directly on the ROC curve.

While it is immediate that this latter semi-parametric approach is more flexible than the fully-parametric model, semi-parametric models may still lose particular robustness properties under model misspecification. Consider the situation of comparing the discriminatory abilities of two potential markers that in truth have the same ROC curve but do not necessarily have the same distribution in the underlying population. Under misspecification of the parametric ROC form, we will likely predict the individual ROC curves and some summary of the curves, such as AUC, to be dissimilar from the true underlying curve or value. This by itself will not pose a problem when testing the strong null of $R O C_{1}(t)=R O C_{2}(t) \forall$ $t$, provided the misestimation is consistent for any given true ROC curve. If a regression method is able to consistently estimate exact equivalence, regardless of whether the true ROC is estimated, it is immediate that any two functionals can be estimated. This situation is highlighted in panel (a) of Figure 1, where two fitted ROC curves overlap even though 
the true ROC curve is not correctly predicted. But the degree of misestimation may differ, and we could incorrectly estimate the two ROC curves to be nonequivalent. In this latter situation, the equivalence of any two functionals can not in general be correctly detected. The situation where the degree of misestimation differs for two marker is shown in panel (b) of Figure 1.

(a)

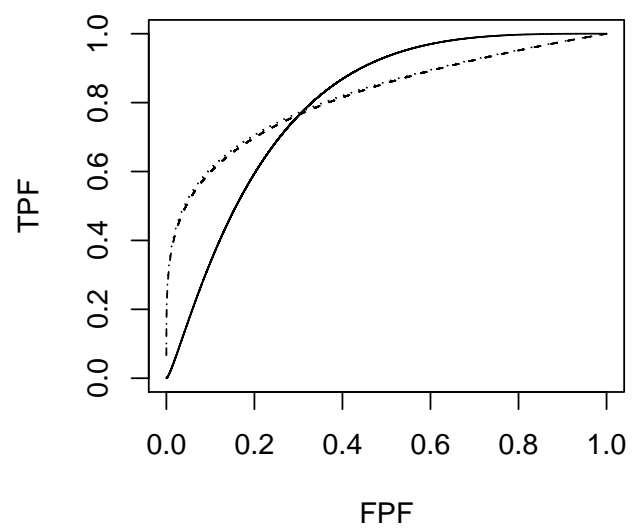

(b)



Figure 1: Fitted curves for two markers that have the same true ROC curve (solid). (a) The degree of misestimation is the same for the two markers and the two fitted ROC curves overlap. (b) The degree of misestimation differs for the two fitted curves.

In this paper, we investigate the ability of various regression methods to test the strong null for two markers over the entire ROC curve in the presence of model misspecification. To illustrate these issues, we focus on the power ROC curve, defined as $R O C(t)=t^{\exp \{\theta\}}$. While not common in ROC modeling, the power curve is attractive in this setting as it is a single parameter family of curves and has various parametric and semi-parametric models readily available for estimation. For instance, the power ROC curve can either be estimated by parametrically modeling the marker distributions using exponential or Weibull regression, by semi-parametrically modeling the marker distributions using the estimating equation derived by the usual partial likelihood methods in proportional hazards time-to-event analyses, 
or by parametrically modeling the ROC curve directly using the estimating equations proposed by Pepe (2003). When the necessary assumptions hold, all three methods consistently estimate the same ROC curve. But when the underlying assumptions are violated, the degree of robustness for the three methods may vary widely. In this paper, we investigate the robustness properties of these methods under misspecification of the marker distribution and/or parametric form of the ROC curve.

\section{Estimation using exponential regression}

Fully parametric approaches to modeling the marker distribution that lead to the power ROC curve are the exponentional and Weibull regression models. In this paper, we will focus on exponential regression. For this approach, define the survival function in the healthy and disease populations to be $S_{\bar{D}}(y)=e^{-\lambda_{1} y}$ and $S_{D}(y)=e^{-\lambda_{2} y}$, respectively. This induces the power ROC curve through $R O C(t)=S_{D}\left(S_{\bar{D}}^{-1}(t)\right)=e^{\left(\lambda_{2} / \lambda_{1}\right) \log (t)}=t^{\exp \{\theta\}}$, where $\theta=\log \left(\lambda_{2} / \lambda_{1}\right)$. The maximum likelihood estimate (MLE) for the two rates are $\hat{\lambda}_{1}=1 / \bar{Y}_{\bar{D}}$ and $\hat{\lambda}_{2}=1 / \bar{Y}_{D}$; therefore, the MLE of the parameter in the power ROC curve is $\hat{\theta}=\log \left(\frac{\bar{Y}_{\bar{D}}}{\bar{Y}_{D}}\right)$.

\section{Estimation using Cox regression}

The semiparametric model of the marker distributions that leads to the power ROC curve is Cox proportional hazards regression. Under the proportional hazards assumption, the corre-

spondence between the healthy and diseased survival functions is $S_{D}(y)=\left(S_{\bar{D}}(y)\right)^{\exp \{\theta\}}$. The power ROC curve is then induced through $R O C(t)=S_{D}\left(S_{\bar{D}}^{-1}(t)\right)=\left(S_{\bar{D}}\left(S_{\bar{D}}^{-1}(t)\right)\right)^{\exp \{\theta\}}=$ $t^{\exp \{\theta\}}$.

For this regression approach, let $Y_{i}, i \in 1 \ldots N$, denote marker values in the pooled healthy and diseased sample, and let $\mathbf{x}_{i}$ denote the design matrix. Without censoring or ties, the partial likelihood based estimating equation is 


$$
\mathcal{U}(\theta)=\sum_{i=1}^{N}\left(\mathbf{x}_{i}-\frac{\sum_{j=1}^{N} I\left[Y_{j} \geq Y_{i}\right] \mathbf{x}_{j} e^{\mathbf{x}_{j}^{T} \theta}}{\sum_{j=1}^{N} I\left[Y_{j} \geq Y_{i}\right] e^{\mathbf{x}_{j}^{T} \theta}}\right)=0 .
$$

For this paper, we focus on the estimating equation without covariates. It can be rewritten in terms of the empirical ROC curve $R \hat{O} C(t)$ as

$$
\mathcal{U}(\theta)=\frac{1}{N_{D}} \sum_{i=1}^{N_{D}} \frac{r R \hat{O} C^{-1}\left(u_{i}\right)}{u_{i} e^{\theta}+r R \hat{O} C^{-1}\left(u_{i}\right)}-\frac{1}{N_{\bar{D}}} \sum_{i=1}^{N_{\bar{D}}} \frac{R \hat{O} C\left(t_{i}\right) e^{\theta}}{r^{-1} R \hat{O} C\left(t_{i}\right) e^{\theta}+t_{i}},
$$

where $r=\frac{N_{\bar{D}}}{N_{D}}, u_{i}=\frac{N_{D}-i+1}{N_{D}}$ and $t_{i}=\frac{N_{\bar{D}}-i+1}{N_{\bar{D}}}$. As $N_{D} \rightarrow \infty$ and $N_{\bar{D}} \rightarrow \infty$ but the ratio of sample sizes, $r$, stays constant, empirical process theory provides $R \hat{O} C(t) \rightarrow R O C(t)$, $R \hat{O} C(t)^{-1} \rightarrow R O C^{-1}(t)$, and the estimating equation converges to

$$
0=\int_{0}^{1} \frac{r R O C^{-1}(u)}{u e^{\theta}+r R O C^{-1}(u)} d u-\int_{0}^{1} \frac{R O C(t) e^{\theta}}{r^{-1} R O C(t) e^{\theta}+t} d t
$$

\section{Estimation using ROC-GLM}

The ROC-GLM method uses a parametric model of the ROC curve which induces a semiparametric relationship between the two marker distributions. This approach focuses on minimizing a weighted difference between a nonparametric estimate of the ROC curve and a specified parametric ROC curve across an interval of evenly spaced false positive fractions (FPFs). Since the aim here is to estimate the entire ROC curve, let the interval $T$ be a vector of evenly spaced FPFs across the range $(0,1)$. Let $N_{t}$ denote the total number of 
points in $T$ and let $k$ denote the index of $T$.

Define an invertible link function $g()$ so that $g\left(R O C\left(t_{k}\right)\right)=\alpha_{0}\left(t_{k}\right)+\vec{\theta}^{T} \vec{\alpha}\left(t_{k}\right)$ is linear in its parameters and $R O C\left(t_{k}\right)$ is monotonic and increasing over the interval $(0,1)$. Furthermore, when choosing the link function, the specified curve must be a proper ROC curve as $t_{k} \rightarrow 1^{-}$ and $t_{k} \rightarrow 0^{+}$. To this end, we introduce the offset $\alpha_{0}\left(t_{k}\right)$, which is shown below to be a requirement for certain parameterizations of the ROC curve.

Using the weighting function $w(t)=\frac{\frac{d}{d l} R O C(l)}{R O C(l)(1-R O C(l))}$, where $l=\alpha_{0}\left(t_{k}\right)+\theta \alpha\left(t_{k}\right)+\beta X$, the general form for the ROC-GLM estimating equation is

$$
\mathcal{U}(\theta, \beta)=\sum_{k=1}^{N_{t}} \sum_{i=1}^{N_{D}}\left(\alpha\left(t_{k}\right), X_{i}\right)^{T} w\left(t_{k}\right)\left[\hat{U}_{i, t}-g^{-1}\left(\alpha_{0}\left(t_{k}\right)+\theta \alpha\left(t_{k}\right)+\beta X_{i}\right)\right]
$$

where the mean of $\hat{U}_{i, t_{k}}$ over the diseased population is a nonparametric estimate of the ROC curve at the FPF $t_{k}: \frac{1}{N_{D}} \sum_{i=1}^{N_{D}} \hat{U}_{i, t_{k}}=R \hat{O} C\left(t_{k}\right)$.

When applying this method to the power ROC curve family, the required link is the complementary $\log -\log$ function, $g(x)=\log (-\log (x))$, so that $\left.g\left(R O C\left(t_{k}\right)\right)=\theta+\log \left(-\log t_{k}\right)\right)+$ $X_{i} \beta$. Here $\alpha\left(t_{k}\right)=1, w\left(t_{k}\right)=\frac{\log t_{k}^{\theta+X_{i} \beta}}{\left(1-t_{k}^{\theta+X_{i} \beta}\right)}$, and $\alpha_{0}\left(t_{k}\right)=\log \left(-\log t_{k}\right)$ the required offset. The complementary log-log function must be used here over the single log, since in the latter case the ROC would then be defined as $R O C\left(t_{k}\right)=\exp \left\{e^{\theta} \log \left(t_{k}\right)+X_{i} \beta\right\}$; this quantity may not be defined as a proper ROC curve when $t_{k} \rightarrow 1^{-}$.

Without covariates, the estimating equation for the power curve ROC-GLM is then

$$
\begin{aligned}
\mathcal{U}(\theta) & =\sum_{k=1}^{N_{t}} \sum_{i=1}^{N_{D}} \frac{\log t_{k}^{\theta^{\theta}}}{\left(1-t_{k}^{e^{\theta}}\right)}\left[\hat{U}_{i t}-t_{k}^{e^{\theta}}\right]=0 \\
& =\frac{1}{N_{t}} \sum_{k=1}^{N_{t}} \frac{\log t_{k}^{e^{\theta}}}{\left(1-t_{k}^{e^{\theta}}\right)}\left[R \hat{O} C\left(t_{k}\right)-t_{k}^{e^{\theta}}\right]=0
\end{aligned}
$$

If $T$ is defined as the vector $T=\left\{1 / N_{\bar{D}}, \ldots,\left(N_{\bar{D}}-1\right) / N_{\bar{D}}\right\}$ and $N_{D}, N_{\bar{D}} \rightarrow \infty$, the ROC- 
GLM estimating equation converges to

$$
\int_{0}^{1} \frac{\log t^{e^{\theta}}}{\left(1-t^{e^{\theta}}\right)}\left[R O C(t)-t^{e^{\theta}}\right] d t=0
$$

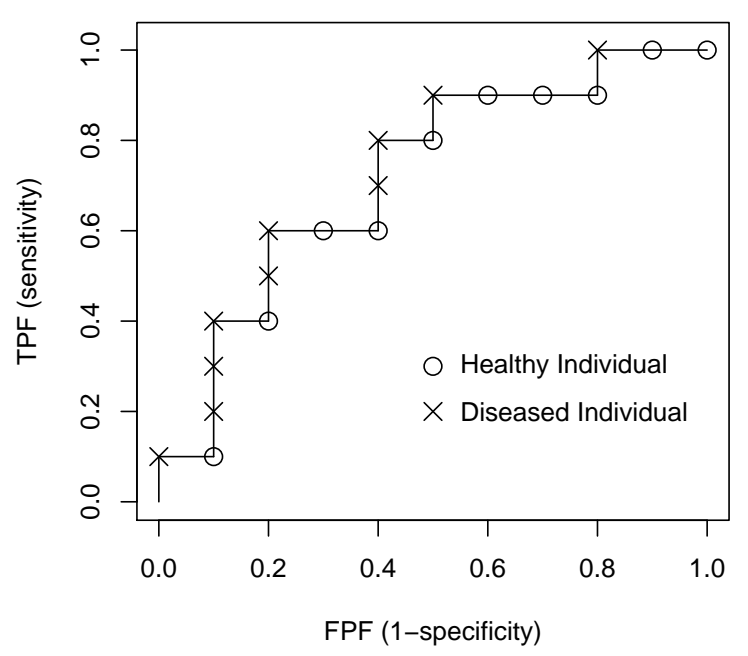

Figure 2: An empirical ROC curve for 10 individuals in both the healthy and diseased samples. Each mark $(\times, \circ)$ corresponds to the empirical FPF and TPF when the test threshold is identical to an observed marker value.

For the nonparametric estimate of the ROC curve, $R \hat{O} C\left(t_{k}\right)=\frac{1}{N_{D}} \sum_{i=1}^{N_{D}} \hat{U}_{i, t_{k}}$, Pepe (2003) used the indicator function $\hat{U}_{i, t_{k}}=I\left[\hat{S}_{\bar{D}}\left(Y_{D i}\right) \leq t_{k}\right]$. This can be expanded to a class of nonparametric estimates $\hat{U}_{i, t_{k}}=I\left[\hat{S}_{\bar{D}}\left(Y_{D i}\right)<t_{k}\right]+\alpha I\left[\hat{S}_{\bar{D}}\left(Y_{D i}\right)=t_{k}\right]$, where $\alpha \in[0,1]$. The estimates corresponding to different values of $\alpha$ can be viewed in Figure 2. For a given FPF $t_{k}$ corresponding to vertical jump on the empirical curve, any TPF along the vertical segment would be a valid estimate of ROC curve at $t_{k}$. A value of $\alpha=1$ corresponds to assigning the maximum TPF for the vertical segment, as adopted by Pepe, while $\alpha=0$ corresponds to assigning the minimum TPF. Other options include assigning the midpoint of the segment, or some weighted combination of the estimated curves using $\alpha=1$ and $\alpha=0$ 
as suggested by Alonzo and Pepe (2002).

While most values of $\alpha$ will be consistent estimates of the ROC curve, some may provide systematically less bias with smaller samples. Consider ROC-GLM estimates of the power ROC curve when the true value of $\theta=-1.2$, and there are 300 individuals in the healthy and diseased samples both of which have an exponential marker distribution. In 5,000 simulated studies, the mean and standard deviation of parameter estimates and corresponding $z$-statistics were calculated when $\alpha=0, \alpha=1, \alpha=0.5$. As shown in Table 1 , the average $\hat{\theta}$ depart from the true value depending on the choice of $\alpha$, and these deviations are large based on the $z$-statistic. For the remainder of this article, we will use $\alpha=0.5$, as it provides smaller bias.

Table 1: ROC-GLM estimates of $\theta$ for three possible $\hat{U}_{i, t_{k}}$ options.

\begin{tabular}{|c|cc|}
\hline$\hat{U}_{i, t_{k}}$ & Mean (SD) & $z$-statistic \\
\hline$\alpha=0$ & $-1.194(0.106)$ & 4.00 \\
$\alpha=0.5$ & $-1.203(0.107)$ & -1.98 \\
$\alpha=1$ & $-1.212(0.107)$ & -7.93 \\
\hline
\end{tabular}

\section{Comparing the three regression methods}

We are interested in the ability of the regression methods to contrast two or more markers when the power curve is the correct specification of the true curve in the population and when it is not the true underlying curve. Within correct and incorrect specification of the ROC curve, we further investigate whether the parameter estimates are dependent on the marker distribution in the population.

Correct specification of the ROC curve

Under the power ROC curve, the inverse survival function in the diseased population is specified knowing the healthy marker distribution through $S_{D}^{-1}(t)=S_{\bar{D}}^{-1}\left(t^{\exp \{-\theta\}}\right)$. Using this relationship, data were generated when the distribution in the healthy population was Exponential $(\lambda=1)$, Normal $(\mu=150, \sigma=5)$, and Log-Normal $(\mu=3, \sigma=1)$ and the true 
value of $\theta=-1.2$. Fixing the total sample size at 600 , we considered three situations when the ratio of diseased to healthy was either $1: 1,1: 5$ or 5:1. Over 5,000 replications of the data generating process, the mean and standard deviation of $\hat{\theta}$ estimates were calculated.

For estimates using ROC-GLM, we considered three different vectors of FPFs. These included one based on the empirical distribution using the maximal set of FPFs $T_{\max }=$ $\left\{1 / N_{\bar{D}}, \ldots,\left(N_{\bar{D}}-1\right) / N_{\bar{D}}\right\}$, and two other vectors using 10 and 100 evenly spaced FPF points, $T_{10}$ and $T_{100}$, respectively.

Simulation results are presented in Table 2. The exponential regression model provides unbiased and efficient estimates when the marker distribution is exponential, but the estimates deviate from the true value when the marker is no longer exponential. The estimated ROC curves under the three marker distributions are shown in Figure 3(a). While the estimated ROC curve for an exponential healthy distribution appears identical to the true ROC, the curves for the two other distributions are considerably different from the true curve. This approach appears to estimate the same parameter value for any ratio of sample sizes.

Under any combination of marker distribution and sample size ratio, the Cox and ROCGLM methods provide similar estimates of $\theta$. As the most efficient rank-based method under proportional hazards, standard errors from the Cox model appear to be consistently smaller than from ROC-GLM. Estimates from both methods appear slightly negatively biased. The efficiency of the ROC-GLM estimates appears to be dependent upon the choice of T. Standard errors for the estimates of $\theta$ from $T_{\max }$ are always smaller than either $T_{100}$ or $T_{10}$. The $T_{\max }$ and $T_{100}$ vectors are equal when the diseased to healthy ratio is 500:100. The standard errors are consistently largest for the $T_{10}$ vector.

Misspecification of the ROC curve

Unlike the construction above, data here were generated when the true underlying ROC curve was binormal. The binormal ROC curve is parameterized as $R O C(t)=S_{D}\left(S_{\bar{D}}^{-1}(t)\right)=$ $\Phi\left(a+b \Phi^{-1}(t)\right)$, where $a=\frac{\mu_{D}-\mu_{\bar{D}}}{\sigma_{D}}$ and $b=\frac{\sigma_{\bar{D}}}{\sigma_{D}}$. Using this relationship, and a specified 
(a)

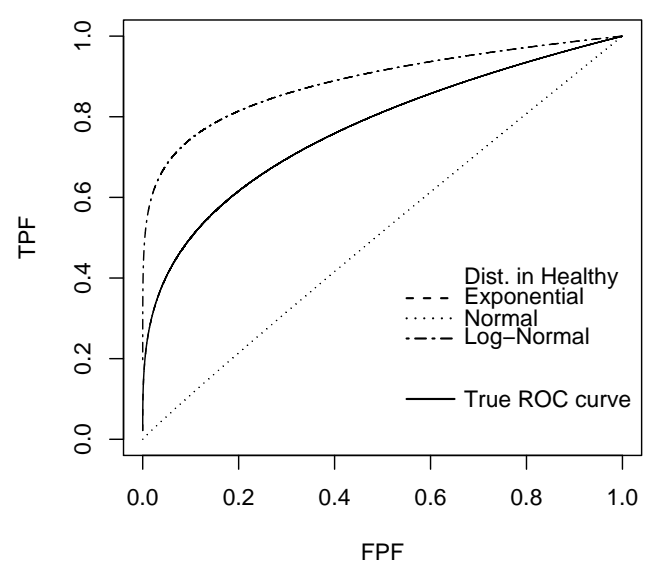

(b)

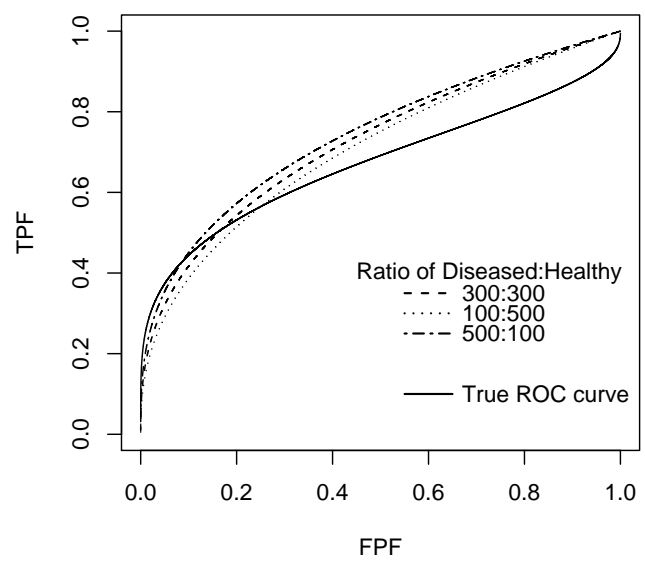

Figure 3: (a) Exponential regression estimates of $\theta$ when the true ROC curve is a power curve. The estimated curve when the healthy marker distribution is exponential overlaps the true ROC curve. There were 300 individuals in both the healthy and diseased samples. (b) Estimated ROC curves from Cox regression when the true ROC curve is binormal and the healthy marker distribution is exponential. The estimate $\hat{\theta}$ was the average over 5,000 replications.

marker distribution in the healthy population, the inverse survival function in the diseased population is defined as $S_{D}^{-1}(t)=S_{\bar{D}}^{-1}\left(\Phi\left(\frac{\Phi^{-1}(t)-a}{b}\right)\right)$. Data were again generated in the healthy population from exponential, normal and log-normal distributions, and the true value for $a$ and $b$ were both 0.5 . The number of replications and sample sizes ratios were the same as before. Since the true ROC curve is not a power curve, there is no true value of $\theta$.

Results from this simulation are provided in Table 3. Exponential regression again provides different estimates of $\theta$ depending on the marker distribution in the healthy; but, for a specified healthy distribution, this approach provides similar estimates across different ratios of sample sizes. In constrast to this, Cox regression provides similar estimates for a fixed sample size ratio; but as this ratio is varied, this model estimates different values for the parameter. Figure 3(b) displays the estimated ROC curves using Cox regression when the sample size ratio is varied. 
Unlike exponential or Cox regression, ROC-GLM estimates similar values for $\theta$ for any combination of healthy marker distribution and ratio of sample sizes. Again, the standard error for the estimates appear to vary slightly depending on the choice of T. Parameter estimates from $T_{10}$ differ from the other two vectors, potentially due to the degree of curvature in the chosen binormal curve.

\section{Discussion}

There are various statistical regression models available to estimate the single parameter in the power ROC curve. These parametric or semi-parametric methods focus on either modeling the marker distributions, which induce a ROC curve, or direct modeling of the parametric form of the ROC curve. In this paper, we investigated the ability of these methods to contrast two or more markers under correct and incorrect specification of the ROC curve with various underlying marker distributions.

Fully parametric exponential regression provides efficient estimates under correct specification of the marker distribution, but the ability to provide consistent estimates remains dependent on the marker distribution.

The two other regression models attempt to relax the strong distributional assumptions inherent in fully-parametric models. Cox regression provides similar estimates for various marker distributions, as long as the true ROC curve follows a power curve. When the parametric form was not a power curve, the dependence of the estimating equation on the ratio of the two sample sizes is apparent; the model estimates varying values of the parameter depending this ratio.

An alternative regression model, ROC-GLM, instead focuses on modeling the parametric form of the ROC curve. The estimating equation for this method does not depend on characteristics of the combined healthy and diseased samples. Therefore, this approach was able to consistently provide similar contrasts of the markers independent of whether the 
parametric ROC form was correctly specified. The choice of the FPF vector in ROC-GLM did appear to influence efficiency as previously reported in Alonzo and Pepe (2002). While a large vector may influence the computational complexity of this approach, we feel that the majority of users will likely use the vector based on the empirical healthy distribution.

In summary, ROC-GLM appears to be more robust to model misspecification when testing the strong null. ROC-GLM was able to consistently test the equality of ROC curves under all marker distributions or misspecification of the parametric ROC form. The dependence on the ratio of sample sizes for the partial likelihood estimating equation in proportional hazards regression, and its impact on parameter misestimation, suggest difficulty in casecontrol studies where the prevalence of disease may be chosen by study design. We found that when ROC inference is the goal using estimating equations based on the parametric form of the ROC curve rather than the marker distribution is important. The weighted difference basically between a nonparametric estimate $\hat{\theta}^{N P}$ and a chosen semiparametric form $\theta^{S P}$ of the ROC curve

$$
\mathcal{U}(\theta)=\sum_{i}^{N} w_{i}\left(\hat{\theta}_{i}^{N P}-\theta_{i}^{S P}\right)
$$

for ROC-GLM appears to be the source of the better behavior. 
Table 2: Comparison of exponential, Cox proportional hazards and ROC-GLM estimates of $\theta$ when the true ROC curve is a power curve and $\theta=-1.2$. Estimates were calculated as the mean and standard deviation of 5,000 replications.

\begin{tabular}{|cr|ccc|}
\hline & & \multicolumn{3}{|c|}{ Ratio of Diseased:Healthy } \\
Regression Type & $F_{\bar{D}}$ & $300: 300$ & $500: 100$ & $100: 500$ \\
\hline Exponential & Exp. & $-1.198(0.082)$ & $-1.204(0.109)$ & $-1.195(0.109)$ \\
& Normal & $-0.044(0.003)$ & $-0.044(0.004)$ & $-0.044(0.005)$ \\
& Log-Norm. & $-2.057(0.205)$ & $-2.073(0.203)$ & $-2.026(0.318)$ \\
\hline Cox PH & & $-1.202(0.095)$ & $-1.206(0.117)$ & $-1.202(0.125)$ \\
& $-1.202(0.093)$ & $-1.206(0.117)$ & $-1.202(0.124)$ \\
& $-1.203(0.094)$ & $-1.207(0.120)$ & $-1.201(0.127)$ \\
\hline ROC-GLM $T_{\max }$ & $-1.203(0.107)$ & $-1.204(0.122)$ & $-1.208(0.156)$ \\
& $-1.202(0.104)$ & $-1.203(0.122)$ & $-1.207(0.155)$ \\
& $-1.204(0.105)$ & $-1.204(0.124)$ & $-1.208(0.157)$ \\
\hline ROC-GLM $T_{10}$ & $-1.204(0.115)$ & $-1.204(0.130)$ & $-1.210(0.170)$ \\
& & $-1.202(0.112)$ & $-1.202(0.130)$ & $-1.209(0.169)$ \\
& $-1.204(0.113)$ & $-1.203(0.131)$ & $-1.211(0.172)$ \\
\hline ROC-GLM $T_{100}$ & $-1.203(0.107)$ & $-1.204(0.122)$ & $-1.208(0.157)$ \\
& $-1.202(0.104)$ & $-1.203(0.122)$ & $-1.209(0.157)$ \\
& $-1.204(0.105)$ & $-1.204(0.124)$ & $-1.208(0.159)$ \\
\hline
\end{tabular}


Table 3: Comparison of exponential, Cox proportional hazards and ROC-GLM estimates of $\theta$ when the true ROC curve is binormal. Estimates were calculated as the mean and standard deviation of 5,000 replications.

\begin{tabular}{|c|c|c|c|c|}
\hline \multirow[b]{2}{*}{ Regression Type } & \multirow[b]{2}{*}{$F_{\bar{D}}$} & \multicolumn{3}{|c|}{ Ratio of Diseased:Healthy } \\
\hline & & $300: 300$ & $100: 500$ & $500: 100$ \\
\hline \multirow[t]{3}{*}{ Exponential } & Exp. & $-1.197(0.092)$ & $-1.201(0.115)$ & $-1.190(0.131)$ \\
\hline & Normal & $-0.033(0.004)$ & $-0.033(0.004)$ & $-0.033(0.007)$ \\
\hline & Log-Norm. & $-2.448(0.294)$ & $-2.470(0.268)$ & $-2.383(0.441)$ \\
\hline \multirow[t]{3}{*}{ Cox PH } & & $-0.969(0.092)$ & $-0.885(0.095)$ & $-1.060(0.138)$ \\
\hline & & $-0.969(0.091)$ & $-0.888(0.095)$ & $-1.058(0.139)$ \\
\hline & & $-0.970(0.090)$ & $-0.886(0.093)$ & $-1.059(0.140)$ \\
\hline \multirow{3}{*}{\multicolumn{2}{|c|}{ ROC-GLM $T_{\max }$}} & $-0.782(0.095)$ & $-0.778(0.098)$ & $-0.788(0.153)$ \\
\hline & & $-0.782(0.094)$ & $-0.780(0.097)$ & $-0.788(0.150)$ \\
\hline & & $-0.784(0.093)$ & $-0.778(0.095)$ & $-0.788(0.155)$ \\
\hline \multirow[t]{3}{*}{ ROC-GLM $T_{10}$} & & $-0.741(0.098)$ & $-0.739(0.100)$ & $-0.747(0.159)$ \\
\hline & & $-0.741(0.097)$ & $-0.742(0.100)$ & $-0.747(0.155)$ \\
\hline & & $-0.742(0.096)$ & $-0.740(0.097)$ & $-0.747(0.160)$ \\
\hline \multirow[t]{3}{*}{ ROC-GLM $T_{100}$} & & $-0.779(0.095)$ & $-0.778(0.098)$ & $-0.784(0.153)$ \\
\hline & & $-0.779(0.094)$ & $-0.780(0.097)$ & $-0.784(0.150)$ \\
\hline & & $-0.780(0.093)$ & $-0.778(0.095)$ & $-0.784(0.155)$ \\
\hline
\end{tabular}




\section{References}

[1] Zweig, M.H. and Campbell, G. (1993). Receiver-Operating Characteristic (ROC) Plots: A Fundamental Evaluation Took in Clinical Medicine. Clinical Chemistry 39, 561-577.

[2] Pepe, M.S. (1998). Three Approaches to Regression Analysis of Receiver Operating Characteristic Curves for Continuous Test Results. Biometrics 54, 124-135.

[3] Alonzo, T.A. and Pepe, M.S. (2002). Distribution-free ROC analysis using binary regression techniques. Biostatistics 3, 421-432.

[4] Pepe, M.S. (2003). The Statistical Evaluation of Medical Tests for Classification and Prediction. Oxford University Press, New York. 